\title{
Positron-Annihilation Momentum Profiles in Aluminum: Core Contribution and the Independent-Particle Model
}

\section{Citation}

Lynn, K. G., MacDonald, J. R., Boie, R. A., Feldman, L. C., Gabbe, J. D.; Robbins, M. F., Bonderup, E., Golovchenko, J. 1977. Positron-Annihilation Momentum Profiles in Aluminum: Core Contribution and the Independent-Particle Model. Physical Review Letters 38, no. 5: 241-244 . doi:10.1103/PhysRevLett.38.241

\section{Published Version}

doi:10.1103/PhysRevLett.38.241;http://dx.doi.org/10.1103/PhysRevLett.38.241

\section{Permanent link}

http://nrs.harvard.edu/urn-3:HUL.InstRepos:29407066

\section{Terms of Use}

This article was downloaded from Harvard University's DASH repository, and is made available under the terms and conditions applicable to Other Posted Material, as set forth at http:// nrs.harvard.edu/urn-3:HUL.InstRepos:dash.current.terms-of-use\#LAA

\section{Share Your Story}

The Harvard community has made this article openly available.

Please share how this access benefits you. Submit a story. 


\title{
Positron-Annihilation Momentum Profiles in Aluminum: Core Contribution and the Independent-Particle Model
}

\author{
K. G. Lynn* \\ Brookhaven National Laboratory, $\dagger$ Upton, New York 11973 \\ and \\ J. R. MacDonald, $\ddagger$ R. A. Boie, L. C. Feldman, J. D. Gabbe, and M. F. Robbins \\ Bell Laboratories, Murray Hill, New Jersey 07974 \\ and \\ E. Bonderup and J. Golovchenko \\ Institute of Physics, University of Aarhus, 8000 Aarhus C, Denmark \\ (Received 17 August 1976)
}

\begin{abstract}
The momentum profiles from positron annihilation with core electrons in $\mathrm{Al}$ have been observed with a recently developed two-detector Doppler-broadening technique. Independent-particle-model (IPM) calculations are in good agreement with the experimental results beyond twice the Fermi momentum and suggest the absence of strong positron-electron correlation effects in this region. Comparisons of calculations with properly normalized momentum profiles indicate that high-momentum core contributions may provide information on the nature of vacancy-type defects and self-trapping effects.
\end{abstract}

This Letter is concerned with positrons annihilating in pure metals after thermalization. In metals a positron goes into an extended Bloch state. In the dominant decay mode of a thermal positron and an electron, two $\gamma$ rays are emitted. Since the annihilating electron possesses momentum, the relative angle between the two photons is slightly less than $\pi$, and the energies are Doppler-shifted. From a measurement of either the distribution in relative angle or the Doppler shift, information about the momentum distribution of the electrons can be obtained. Angular-correlation studies have been very useful for determining electron momenta up to approximately twice the Fermi momentum. ${ }^{1}$

Because of the high potential barriers experienced by a positron in the regions close to nuclei, annihilation with deeply bound core electrons is very improbable as compared to annihilation with outer electrons. Consequently, the very-highmomentum parts of the spectra are of quite low relative intensity, and so far they have not been experimentally accessible because of background and counting-rate problems. In the following we describe how these problems have been overcome such that annihilation with Al $1 s$ electrons is observable.

Information on the annihilation with core electrons is desirable for several reasons. First, to obtain a reliable determination of the momentum distribution of conduction electrons, one must be able to subtract the core contribution from the measured momentum profiles. Second, at sufficiently high momenta the simple independent-particle model (IPM) should suffice for the description of the annihilation. By way of contrast, for annihilation with conduction electrons, one obtains large enhancements above the predictions of the IPM because of the attraction between the positron and the electron under consideration. The high-momentum tails should thus provide a convenient limit for experimental and theoretical investigations of this enhancement effect. Third, having assessed the applicability of the independent-particle model in the high-momentum limit, we expect that comparisons between simple model calculations and experimental momentum distributions, solely from deep-core electrons in samples containing trapping centers, can yield quantitative information on these defects.

To measure the high-momentum regions of the electron distribution, we found it necessary to extend the conventional Doppler-broadening method, which examines only one of the photons from the annihilation process. Our experimental apparatus consists of two $\mathrm{Ge}(\mathrm{Li})$ detectors situated so as to detect both $\gamma$ rays in coincidence from positrons annihilating in the sample under study. ${ }^{2,3}$ Each detector has an efficiency of $10 \%$ and an energy resolution of $\sim 1.6 \mathrm{-keV}$ full width at halfmaximum at $514 \mathrm{keV}$. The resulting energy signals $E_{1}$ and $E_{2}$ are converted to digital form; and the sum and difference of the two energies are calculated by and stored in a computer. 


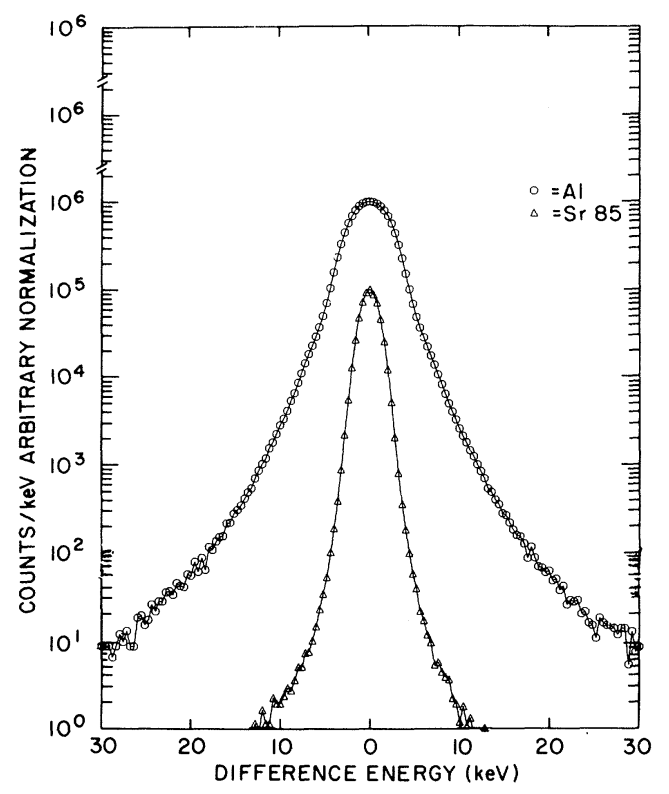

FIG. 1. Experimental Doppler-broadened spectra in the difference energy are shown including data in the sum energy from $\left(2 m_{0} c^{2}+0.4 \mathrm{keV}\right)$ to $\left(2 m_{0} c^{2}-2.0 \mathrm{keV}\right)$ and a similar range of $E_{T}$ for the Sr-85 curve. The Sr85 curve is a measurement of the response of the system.

The sum energy $E_{T}=E_{1}+E_{2}$ equals the total energy of the electron-positron system prior to annihilation, i.e., $E_{T}=2 m_{0} c^{2}-E_{B}$, where $m_{0}$ is the electron rest mass, $c$ the velocity of light, and $E_{B}$ the binding energy of the electron and positron to the solid.

In the electron-positron center-of-mass frame, each photon carries off half of the total energy liberated in the annihilation. In the laboratory frame the energies are Doppler-shifted, and the energy difference $\Delta E$ between the two $\gamma$ rays is

$$
\Delta E=E_{2}-E_{1}=\frac{\overrightarrow{\mathrm{c}} \cdot \overrightarrow{\mathrm{p}}-p^{2} c^{2} / E_{T}}{1-\overrightarrow{\mathrm{c}} \cdot \overrightarrow{\mathrm{p}} / E_{T}} \simeq \overrightarrow{\mathrm{c}} \cdot \overrightarrow{\mathrm{p}} .
$$

Here, $\vec{p}$ is the electron-positron momentum in the laboratory system, and $\vec{c}$ is the velocity of light in the direction of the photon with energy $E_{2}$. The approximation $\Delta E \simeq \overrightarrow{\mathrm{c}} \cdot \overrightarrow{\mathrm{p}}$, which holds for $p c \ll E_{T}$, has been applied to the cases studied here. A measurement of $\Delta E$ thus determines the projection of the momentum $\vec{p}$ along the photon direction. In angular-correlation experiments, a perpendicular component is obtained.

When compared with a single-detector Dopplerbroadening scheme, ${ }^{1}$ there is a great advantage of the two-Ge( $\mathrm{Li})$-detector technique for investigating the very low intensity high-momentum tails. This arises from the fact that in the energy regions of interest the single-detector spectrum is distorted by detection of higher-energy $\gamma$ rays, pulse pile-up difficulties, and incomplete charge collection on the low-energy side of the annihilation peak. A single NaI detector in coincidence with a $\mathrm{Ge}(\mathrm{Li})$ detector will partially reduce background on the higher-energy side of the annihilation peak. ${ }^{4}$ In the present experiment, the coincidence requirement reduces the abovementioned background problems by a factor of more than $10^{4}$, thus making measurements in the very-high-momentum regions feasible. A further advantage is an increase in projected momentum resolution by a factor of $\sqrt{2}$ arising from the use of the Doppler shift of both $\gamma$ rays. Finally, as expected, the momentum spectra are symmetrical around zero.

The signal handling in the two-photon coincidence measurement was done to minimize random coincidences and spurious effects of pulse pileup. The system was digitally stabilized on the centroids of the singles events in each detector to eliminate long-term gain shifts.

The response function of the total system was determined by a pseudocoincidence technique involving $514-\mathrm{keV} \gamma$ rays from $\mathrm{Sr}-85$ detected successively in the two detectors. $\mathrm{Sr}-85$ data were taken at various times during a positron run to determine if any systematic changes occurred in the system response function. The experimental Doppler-shift data and the data for the response function were used, without smoothing, in an iterative deconvolution process similar to that of van Cittert. ${ }^{5}$ This procedure avoids the introduction of systematic errors caused by inadequate functional models. Thus far, the deconvolutions have been carried out only with respect to the variable $\Delta E$.

We have investigated a single-crystal Al sample of purity greater than $99.999 \%$ annealed at three quarters of the absolute melting point for more than 12 hours and slowly cooled. Approximately $50 \mu \mathrm{Ci}$ of $\mathrm{Ge}-68$ was deposited as a thin film on one of the inside surfaces of a two-sample sandwich to minimize the source contribution to the annihilation photopeak. The coincidence data rate was approximately 100 counts per second. The singles rate was 10 times larger, when measured in the photopeak. Total data accumulation time was typically 72 hours.

Figure 1 shows the raw spectra of $\mathrm{Al}$ and the measured response curve for $\mathrm{Sr}-85$. These spectra are plotted as a function of the difference en- 


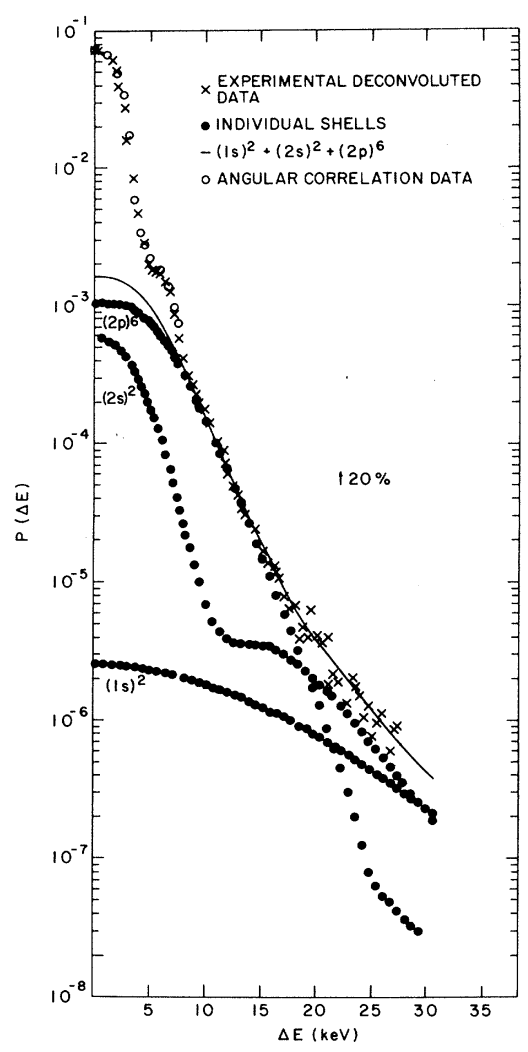

FIG. 2. Comparison of experimental and calculated probability distributions for Al. Wigner-Seitz calculation with overlapping atomic potentials. The different calculations leads only to a $20 \%$ variation in the predicted curves, and the size of these deviations are shown in the figure. Open circles and crosses refer to measurements in Ref. 6 and in this work, respectively; $\tau_{\exp }$ $=175$ psec. $\int_{0}^{\infty} P(\Delta E) d(\Delta E)=\frac{1}{2}$. The Fermi momentum for aluminum corresponds to $\Delta E=3.47 \mathrm{keV}$.

ergy and include all data in the sum energy from $\left(2 m_{0} c^{2}+0.4 \mathrm{keV}\right)$ to $\left(2 m_{0} c^{2}-2.0 \mathrm{keV}\right)$ and a similar range of $E_{T}$ for the Sr-85 pseudocoincidences.

Figure 2 contains the deconvoluted data for $\mathrm{Al}$ in the form of a probability distribution $P(\Delta E)$ with $\int_{-\infty}^{\infty} P(\Delta E) d(\Delta E)=1$. Only positive values of $\Delta E$ are shown. The small oscillations beyond 20 $\mathrm{keV}$ arise from deconvolution of the data which had insufficient statistical accuracy in this region. The figure also shows a comparison with data taken by Hautojärvi ${ }^{6}$ in an angular-correlation apparatus of 1-mrad resolution on high-purity well-annealed $\mathrm{Al}$ single crystals. ( $\Delta E$ of 1 $\mathrm{keV}$ corresponds to $\Delta \theta=1.96$ mrad.) The angular distribution was normalized to our spectra at $\Delta E$ $=0$. In the region of overlap, the agreement is good. A small but significant deviation from 5 to $8 \mathrm{keV}$ is observed. We attribute this to the background problem encountered in angular-correla- tion studies at high momenta. We extracted the Fermi momentum of the conduction electrons by fitting a parabola to the top twenty points of the full Doppler-broadened deconvoluted data. The Fermi momentum determined in this way agrees with the free-electron value to within $2 \% .^{7}$

Since the analysis of the data, to be presented in the following, relies on knowledge of the positron lifetime $\tau$, we measured this quantity for Al. Our value of $175 \mathrm{psec}$ is in good agreement with MacKenzie's experimental value of 170 psec. ${ }^{8}$ This agreement supports our assumption that the sample was free of trapping centers.

The probability $P(\Delta E) d(\Delta E)$ is equal to the ratio of the fractional annihilation rate $\lambda(\Delta E) d(\Delta E)$ to the total annihilation rate $\lambda_{\text {tot }}=\int_{-\infty}^{\infty} \lambda(\Delta E) d(\Delta E)$. Similarly, the annihilation probability $P_{i}(\Delta E)$ with an electron in the $i$ th shell is given by

$$
P_{i}(\Delta E)=\lambda_{i}(\Delta E) / \lambda_{\text {tot }}=\tau \lambda_{i}(\Delta E) .
$$

From the independent-particle model, we have calculated the functions $\lambda_{i}(\Delta E)$ and the momentum distribution, $P_{\boldsymbol{i}}(\Delta E)$, for positrons annihilating with core electrons $i=1 s, 2 s$, and $2 p$. The relevant formulas can, for instance, be found in the review paper by West. ${ }^{1}$ Since the emphasis in this investigation is on the high-momentum tails from core electrons, we do not attempt to calculate $\tau$, which is dominated by the contribution from conduction electrons. Insufficient accuracy in this quantity, which is difficult to calculate, ${ }^{1}$ would mask the comparison between theory and experiment for the core electrons.

In the calculations, the electrons are represented by atomic orbitals. The overlap between positron and electron wave functions is only appreciable in regions close to nuclei, where potentials are well known. Consequently, in the important regions the shape of the positron wave function can be obtained quite accurately from numerical integration of the Schrödinger equation. However, the normalization of the positron wave function in the region of overlap is sensitive to details far away from nuclei, where the positron is found with probability close to one. This effect is illustrated by the following examples: A change in the potential from a single atomic potential to a sum of potentials from neighboring atoms leads to an increase in the calculated annihilation rates of the order of $15 \%$. Similarly, we increase the annihilation rates by $\sim 20 \%$ by going from the Wigner-Seitz model ${ }^{9}$ in which the lattice structure of the specimen is neglected, to the augmented-plane-wave model, ${ }^{10}$ 
known from band-structure calculations. While, at present, we do not argue that any of these calculations is superior to the others, we take the above fractional differences as indicative of the sensitivity of the core annihilation rates to details in the positron wave function far away from nuclei. These details should also be sensitive to the presence of vacancy-type defects and comparisons between experiments and simple model calculations may serve to characterize the type of these defects.

Figure 2 contains the calculated distributions $\boldsymbol{P}_{\boldsymbol{i}}(\Delta E)$ for the configurations $(1 s)^{2},(2 s)^{2}$, and $(2 p)^{6}$, as well as the sum over these distributions. The results are from the Wigner-Seitz model with overlapping atomic potentials. As mentioned in the beginning, the IPM should, if anything, result in an underestimate of annihilation rates. From Fig. 2, it is observed that the WignerSeitz model with overlapping atomic potentials leads to very good agreement with the experimental data already at twice the Fermi momentum. At lower momenta, the importance of the electron-positron correlations and contributions from outer electrons become significant, and they raise the data above the theoretical curve. Note that for the most highly Doppler-shifted events $(\Delta E \approx 27 \mathrm{keV})$, significant contributions are coming from the Al $1 s$ electrons.

Even including the slight difference between the various models mentioned above, we markedly disagree with the predictions of Carbotte and Salvadori ${ }^{11}$ according to which, because of enhancement effects, the experimental results should be almost a factor of 3 above the independent-particle-model calculations for $2 s$ and $2 p$ electrons in Al. The Carbotte-Salvadori result seems difficult to understand. The electron densities in the regions occupied by core electrons are very high. If the electrons are considered as a Fermi gas in the spirit of the Thomas-Fermi atom, these high densities lead to much smaller enhancement factors. In fact, from the results of Kahana ${ }^{12}$ one obtains an enhancement of only a few tens of percent.

The authors wish to acknowledge many stimulating conversations with W. L. Brown. We also wish to thank A. N. Goland, S. Kahana, D. N. Lowy, A. P. Mills, Jr., and P. Mauger for useful discussions.

*Resident visitor at Bell Laboratories, Murray Hill, N. J. 07974.

†Work partly supported by the U. S. Energy Research and Development Administration.

$\$$ Present address: Department of Physics, University of Guelph, Guelph, Ont., Canada.

${ }^{1}$ R. N. West, Adv. Phys. 22, 263 (1973).

${ }^{2}$ J. R. MacDonald, R. A. Boie, L. C. Feldman, M. F. Robbins, P. Mauger, and K. G. Lynn, Bull. Am. Phys. Soc. 24, 580 (1975).

${ }^{3} \mathrm{R}$. Douglas, private communication.

${ }^{4}$ K. G. Lynn and A. N. Goland, Solid State Commun. 18, 1599 (1976).

${ }^{5}$ P. H. van Cittert, Z. Phys. 69, 239 (1931); see also, G. K. Wertheim, J. Electron Spectrosc. $\underline{6}, 239$ (1976).

${ }^{6}$ P. Hautojärvi, Solid State Commun. 11, 1049 (1972).

${ }^{7}$ C. Kittel, Introduction to Solid State Physics (Wiley, New York, 1971).

${ }^{8}$ I. K. MacKenzie, private communication.

${ }^{9}$ S. Berko and J. S. Plaskett, Phys. Rev. 112, 1877 (1958).

${ }^{10}$ T. L. Loucks, Augmented Plane Wave Method (Benjamin, New York, 1967).

${ }^{11} \mathrm{~J}$. P. Carbotte and A. Salvadori, Phys. Rev. 162, 290 (1967).

${ }^{12}$ S. Kahana, Phys. Rev. 117, 123 (1960). 